\title{
Weakly Bound States in Heterogeneous Waveguides: A Calculation to Fourth Order
}

\author{
P. AMORE* \\ Facultad de Ciencias, CUICBAS, Universidad de Colima, Bernal Díaz del Castillo 340, Colima, Colima, Mexico \\ (Received January 23, 2017; in final form September 25, 2017)
}

We have extended a previous calculation of the energy of a weakly heterogeneous waveguide to fourth order in the density perturbation, deriving its general expression. For particular configurations where the second and third orders both vanish, we discover that the fourth order contribution lowers in general the energy of the state, below the threshold of the continuum. In these cases the waveguide possesses a localized state. We have applied our general formula to a solvable model with vanishing second and third orders reproducing the exact expression for the fourth order.

DOI: 10.12693/APhysPolA.132.1351

PACS/topics: trapped modes, bound states, deformed waveguide, perturbation theory

\section{Introduction}

It is nowadays a well-known fact that bound states can appear in infinite waveguides or tubes, in presence of an arbitrarily weak bending or of a local, small, enlargement of its section. This behavior has been proved for general configurations in Refs. [1, 2] and it has been investigated for several specific geometrical configurations. It is impossible to refer to all the different works, but we would like to mention the case of the infinite symmetric cross studied by Schult and collaborators in Ref. [3]. Although Ref. [3] is focused on the study of the quantum mechanical bound states of the symmetric cross, the problem is relevant in many areas of physics, such as acoustics, electromagnetism and fluid dynamics (in this respect, it is important to cite the work by Ursell in Refs. [5, 6] who studied the emergence of trapped modes in a semi-infinite canal of fixed width terminating in a sloping beach).

From a mathematical point of view, one needs to solve the Helmholtz equation on an open, infinite domain, with the Dirichlet boundary conditions at the border. In particular, Bulla and collaborators have considered in Ref. [7] the problem of an infinite homogeneous waveguide on the region

$$
\Omega_{\lambda}=\left\{(x, y) \in \mathbb{R}^{2} \mid 0<y<1+\lambda f(x)\right\},
$$

obeying the Dirichlet boundary conditions at the border, assuming that $f$ is a $C^{\infty}(\mathbb{R})$ function of compact support with $f \geq 0$. In their calculation $\lambda>0$ is a parameter which controls the deformation of the border (particularly the case $\lambda=0$ reduces to a straight waveguide, with a purely continuum spectrum). These authors were able to show that, if $\int_{-\infty}^{\infty} f(x) \mathrm{d} x>0$, there is at least one eigenvalue falling below the continuum threshold. They also obtained the exact expression for the energy of the fundamental mode, to second order in the parameter controlling the deformation. Soon after, Exner and Vugalter [8] studied this problem, when the deformation of the

*e-mail: paolo.amore@gmail.com border averages out, i.e. when $\int_{-\infty}^{\infty} f(x) \mathrm{d} x=0$. Interestingly they found out that under certain conditions it is still possible to have a bound state and that the energy gap scales as the fourth power in $\lambda$.

Recently, the present author and collaborators have studied in Ref. [9] a different but related problem: the case of a infinite straight waveguide containing a small inhomogeneity centered at an internal point (assuming the Dirichlet boundary conditions at the border). In that case, it was proved that, when the heterogeneity corresponds to a locally denser region, the eigenfunction of the ground state becomes localized around the heterogeneity and the corresponding energy falls below the continuum threshold. The calculation of Ref. [9] was carried out using perturbation theory up to third order, using an approach originally proposed by Gat and Rosenstein in Ref. [10] for a different problem. As a matter of fact, the implementation of the perturbative scheme must be done with care, since the naive identification of the unperturbed operator with the negative Laplacian would lead to the appearance of divergent contributions in the coefficients of the perturbative series for the energy of the ground state. The emergence of these (infrared) divergences can be easily understood since the spectrum of $(-\Delta)$ on an infinite strip is continuous and therefore the denominators of the coefficients in the RayleighSchrödinger expansion may become arbitrarily small. To avoid this problem in Ref. [10] a suitable unperturbed operator was used, following the approach of Gat and Rosenstein: the spectrum of this operator contains now a localized state and the continuum, with the energy of the localized state falling below the continuum threshold (the separation between the two depends on a parameter $\beta$ in the unperturbed operator which will be eventually set to zero). In this way one is able to carry out the usual perturbative expansion, obtaining explicit expressions which are finite when $\beta \rightarrow 0^{+}$.

In this paper we have extended the calculation of Ref. [9], obtaining the exact general expression for the energy correction to fourth order in the density perturbation. The greater technical difficulty of the present 
calculation derives both because from the larger number of terms and both from their different nature. Working in our perturbation scheme we find that all the infrared divergent terms (i.e. terms which diverge as $\beta \rightarrow 0^{+}$) potentially contained in $E_{0}^{(4)}$ correctly cancel out, as expected. Moreover, for the case where the second and third order corrections both vanish, we find that there is a non-vanishing fourth order correction to the energy of the fundamental mode, which lowers the energy below the continuum threshold. Since the problem of Bulla et al. [7] may be converted to the problem of an infinite heterogeneous waveguide, using a suitable conformal map, our results also provide an alternative approach to the problems studied in Refs. [7] and [8]. Additionally, our formulae apply as well to the case of infinite heterogeneous and deformed waveguides (in this case the "density" in our formulae would involve both the physical density of the waveguide and the "conformal density" obtained from the mapping), thus allowing to treat more general problems. The fourth order formula obtained in the present paper have been recently applied in Ref. [11] to obtain the behaviour of the lowest eigenvalue of a "broken strip", in the case of infinitesimal bendings, reproducing the numerical results in the literature [12].

The paper is organized as follows: in Sect. 2 we discuss the perturbation theory, and present the general formulae for the energy to fourth order; in Sect. 3 we consider a solvable model, reproducing the exact results to fourth order; in Sect. 4 we present our conclusions.

\section{Perturbation theory}

In a recent paper we have obtained the explicit expression for the energy of the fundamental mode of an infinite, weakly heterogeneous two-dimensional waveguide, up to third order in the density perturbation. It is assumed that the inhomogeneity is small and localized at some internal point of the waveguide. Under these assumptions it is proved that, when the perturbation corresponds to a locally denser material, a bound state, localized at the inhomogeneity appears.

Mathematically, we are considering the Helmholtz equation

$$
(-\Delta) \Psi_{n}(\boldsymbol{x})=E_{n} \Sigma(\boldsymbol{x}) \Psi_{n}(\boldsymbol{x}),
$$

where $|x|<\infty$ and $|y| \leq b / 2$. The solutions obey the Dirichlet boundary conditions at the border

$$
\Psi_{n}(x, \pm b / 2)=0
$$

and $\Sigma(x, y)>0$ for $|x|<\infty$ and $|y| \leq b / 2$.

Expressing the density as $\Sigma(\boldsymbol{x})=1+\sigma(\boldsymbol{x})$, where $\lim _{|x| \rightarrow \infty} \sigma(\boldsymbol{x})=0$, and assuming that $|\sigma(x)| \ll 1$ for $x \in(-\infty, \infty)$, we can perform a perturbative expansion in the density perturbation.

The general formulae for the perturbative corrections to the energy of the fundamental mode up to third order have been derived in Refs. [9] and [13] and read

$$
\begin{aligned}
& E_{0}^{(1)}=-\langle\sigma\rangle \epsilon_{0}, \\
& E_{0}^{(2)}=\langle\sigma\rangle^{2} \epsilon_{0}-\langle\sigma \Omega \sigma\rangle \epsilon_{0}^{2},
\end{aligned}
$$

$$
\begin{aligned}
& E_{0}^{(3)}=-\epsilon_{0}\langle\sigma\rangle^{3}+3\langle\sigma\rangle\langle\sigma \Omega \sigma\rangle \epsilon_{0}^{2}+\epsilon_{0}^{3}(\langle\sigma\rangle\langle\sigma \Omega \Omega \sigma\rangle \\
& \quad-\langle\sigma \Omega \sigma \Omega \sigma\rangle)
\end{aligned}
$$

where

$$
\hat{\Omega} \equiv \sum_{n} \frac{|n\rangle\langle n|}{\epsilon_{n}-\epsilon_{0}}
$$

and $\epsilon_{n}$ and $|n\rangle$ are the eigenvalues and eigenstates of the unperturbed operator ${ }^{\dagger}$.

As we have discussed in Ref. [9], the identification of the unperturbed operator must be done with care, for the case of an infinite waveguide: as a matter of fact, the obvious candidate, corresponding to an infinite, straight and homogeneous waveguide cannot be used, since its spectrum is continuous and the fundamental mode can thus be excited to states which are arbitrarily close in energy. In this case, the perturbative formulae would contain infrared divergences, which would completely spoil the calculation. In a different context Gat and Rosenstein [10] have devised a perturbation scheme that allows to avoid these infrared divergences: in our case this process amounts to use as unperturbed operator

$$
\hat{H}_{0}=-\Delta-2 \beta \delta(x),
$$

where $\beta$ is an infinitesimal parameter to be set to 0 at the end of the calculation.

As discussed in Ref. [9], the basis set of eigenfunctions of $\hat{H}_{0}$ is

$$
\Psi_{p, n}(x, y)=\psi_{n}(y) \otimes \begin{cases}\phi_{o}(x) & \text { ground state } \\ \phi_{p}^{(e)}(x) & \text { even state } \\ \phi_{p}^{(o)}(x) & \text { odd state }\end{cases}
$$

where

$$
\begin{aligned}
& \phi_{0}(x)=\sqrt{\beta} \mathrm{e}^{-\beta|x|}, \\
& \phi_{p}^{(e)}(x)=\sqrt{\frac{2}{p^{2}+\beta^{2}}}(p \cos (p x)-\beta \sin (p|x|)), \\
& \phi_{p}^{(o)}(x)=\sqrt{2} \sin (p x),
\end{aligned}
$$

and

$$
\psi_{n}(y)=\sqrt{\frac{2}{b}} \sin \left[\frac{n \pi}{b}(y+b / 2)\right] .
$$

The eigenvalues of $\hat{H}_{0}$ are $\ddagger$

$$
\begin{aligned}
& \epsilon_{0, n}=-\beta^{2}+\frac{n^{2} \pi^{2}}{b^{2}}, \\
& \epsilon_{p, n}^{(e)}=\epsilon_{p, n}^{(o)}=p^{2}+\frac{n^{2} \pi^{2}}{b^{2}} .
\end{aligned}
$$

We find convenient to introduce the Dirac notation $|0, n\rangle$, $\left|p^{(e)}, n\right\rangle$ and $\left|p^{(o)}, n\right\rangle$ to indicate the eigenstates of $\hat{H}_{0}$.

\footnotetext{
${ }^{\dagger}$ In the following we will adopt the notation $\langle\hat{A}\rangle$ to indicate the expectation value of the operator $\hat{A}$ in the ground state of $\hat{H}_{0}$.

$\ddagger_{\text {Note that }} \epsilon_{0,1}=-\beta^{2}+\frac{\pi^{2}}{b^{2}}<\frac{\pi^{2}}{b^{2}}$ and therefore it is separated from the continuum.
} 
Using the explicit form of the eigenfunctions of $\hat{H}_{0}$ given above, one can work out the perturbative expressions for the energy and, after taking the limit $\beta \rightarrow 0^{+}$, obtain the finite expressions given in Ref. [9]:

$$
\begin{aligned}
& \lim _{\beta \rightarrow 0^{+}} E_{0}^{(1)}=0 \\
& \lim _{\beta \rightarrow 0^{+}} E_{0}^{(2)}=-\frac{\pi^{4}}{b^{6}} \\
& \quad \times\left(\int_{-\infty}^{\infty} \mathrm{d} x \int_{-b / 2}^{b / 2} \mathrm{~d} y \sigma(x, y) \cos ^{2}\left(\frac{\pi y}{b}\right)\right)^{2}, \\
& \lim _{\beta \rightarrow 0^{+}} E_{0}^{(3)}=\frac{2 \pi^{6}}{b^{9}} \\
& \quad \times \int_{-\infty}^{\infty} \mathrm{d} x_{3} \int_{-b / 2}^{b / 2} \mathrm{~d} y_{3} \cos ^{2}\left(\frac{\pi y_{3}}{b}\right) \sigma\left(x_{3}, y_{3}\right) \\
& \quad \times \int_{-\infty}^{\infty} \mathrm{d} x_{1} \int_{-b / 2}^{b / 2} \mathrm{~d} y_{1} \int_{-\infty}^{\infty} \mathrm{d} x_{2} \int_{-b / 2}^{b / 2} \mathrm{~d} y_{2} \\
& \quad \times\left[\left|x_{1}-x_{2}\right| \sigma\left(x_{1}, y_{1}\right) \sigma\left(x_{2}, y_{2}\right) \cos ^{2}\left(\frac{\pi y_{1}}{b}\right)\right. \\
& \quad \times \cos ^{2}\left(\frac{\pi y_{2}}{b}\right)-b \cos \left(\frac{\pi y_{1}}{b}\right) \cos \left(\frac{\pi y_{2}}{b}\right) \sigma\left(x_{1}, y_{1}\right) \\
& \left.\quad \times\left.\sigma\left(x_{2}, y_{2}\right) \mathcal{G}_{2}^{(0)}\left(\boldsymbol{x}_{1}, \boldsymbol{x}_{2}\right)\right|_{\beta=0}\right] .
\end{aligned}
$$

where ${ }^{\S}$

$$
\begin{aligned}
\mathcal{G}_{0}^{(\ell)}\left(\boldsymbol{x}, \boldsymbol{x}^{\prime}\right) & \equiv \int_{0}^{\infty} \frac{\mathrm{d} p}{2 \pi} \frac{\phi_{p}(x) \phi_{p}\left(x^{\prime}\right) \psi_{1}(y) \psi_{1}\left(y^{\prime}\right)}{\left(\epsilon_{p, 1}-\epsilon_{0,1}\right)^{\ell+1}} \\
\mathcal{G}_{1}^{(\ell)}\left(\boldsymbol{x}, x^{\prime}\right) & \equiv \sum_{n=2}^{\infty} \frac{\phi_{0}(x) \phi_{0}\left(x^{\prime}\right) \psi_{n}(y) \psi_{n}\left(y^{\prime}\right)}{\left(\epsilon_{0, n}-\epsilon_{0,1}\right)^{\ell+1}} \\
\mathcal{G}_{2}^{(\ell)}\left(\boldsymbol{x}, \boldsymbol{x}^{\prime}\right) & \equiv \sum_{n=2}^{\infty} \int_{0}^{\infty} \frac{\mathrm{d} p}{2 \pi} \frac{\phi_{p}(x) \phi_{p}\left(x^{\prime}\right) \psi_{n}(y) \psi_{n}\left(y^{\prime}\right)}{\left(\epsilon_{p, n}-\epsilon_{0,1}\right)^{\ell+1}}
\end{aligned}
$$

Before discussing the fourth order, it is worth to comment that, as discussed in [9], a bound state is present only if the condition

$$
\int_{-\infty}^{\infty} \int_{-b / 2}^{b / 2} \sigma(x, y) \cos ^{2} \frac{\pi y}{b} \mathrm{~d} x \mathrm{~d} y>0
$$

is met.

We briefly review the discussion in Ref. [9]: the condition (12) can be derived calculating the Rayleigh quotient

$$
W=\frac{\langle\Psi|(-\Delta)| \Psi\rangle}{\langle\Psi|\Sigma| \Psi\rangle}
$$

using the variational function

$$
\Psi(x, y)=\sqrt{a} \mathrm{e}^{-a|x|} \sqrt{\frac{2}{b}} \sin \frac{n \pi(y+b / 2)}{b}
$$

$\S$ Note that we have changed the notation of Ref. [9] to allow referring to the more general Green functions. and minimizing with respect to the variational parameter $a$ :

$$
a_{\min } \approx \frac{\pi^{2}}{b^{3}} \int_{-\infty}^{+\infty} \int_{-b / 2}^{b / 2} \sigma(x, y) \cos ^{2} \frac{\pi y}{b} \mathrm{~d} x \mathrm{~d} y .
$$

Given that, in order to obtain a bound state, $a$ must be positive, the condition (12) follows. In a similar way, one can derive the expression for the perturbative correction to the energy of the fundamental mode to fourth order; we find

$$
\begin{gathered}
E_{0}^{(4)}=\langle\sigma\rangle^{4} \epsilon_{0}-6\langle\sigma\rangle^{2}\langle\sigma \Omega \sigma\rangle \epsilon_{0}^{2}+\left(2\langle\sigma \Omega \sigma\rangle^{2}\right. \\
\left.+4\langle\sigma\rangle\langle\sigma \Omega \sigma \Omega \sigma\rangle-4\langle\sigma\rangle^{2}\langle\sigma \Omega \Omega \sigma\rangle\right) \epsilon_{0}^{3} \\
-(\langle\sigma \Omega \sigma \Omega \sigma \Omega \sigma\rangle-\langle\sigma \Omega \sigma\rangle\langle\sigma \Omega \Omega \sigma\rangle \\
\left.-2\langle\sigma\rangle\langle\sigma \Omega \Omega \sigma \Omega \sigma\rangle+\langle\sigma\rangle^{2}\langle\sigma \Omega \Omega \Omega \sigma\rangle\right) \epsilon_{0}^{4} .
\end{gathered}
$$

The perturbative expressions written above must be evaluated taking the limit $\beta \rightarrow 0^{+}$at the end of the calculation. For this reason it is convenient to work on the expectation values which appear in the expression and expand them around $\beta=0$.

For example, in the simplest case we have

$$
\langle\sigma\rangle=\beta \int \mathrm{d} x \mathrm{~d} y \mathrm{e}^{-2 \beta|x|}\left(\psi_{1}(y)\right)^{2} \sigma(x, y)=\sum_{n=1}^{\infty} \kappa_{1}^{(n)} \beta^{n} .
$$

The expressions for the remaining expectation values can be found in the arxiv version of the present paper, Ref. [14]. In particular, in Table I the coefficients $\kappa_{n}^{(j)}$ are subdivided into two classes: those which only contain longitudinal contributions (left column) and those which contain both longitudinal and tranverse contributions (right column).

TABLE I

Coefficients appearing in the expression of the energy of the fundamental mode up to fourth order in perturbation theory. The coefficients on the right side contain contributions also from the transversal modes.

\begin{tabular}{ccc|cc}
\hline \hline & $\|$ & & \multicolumn{2}{|c}{$\|+\perp$} \\
\hline$\kappa_{1}^{(1)}$ & $\kappa_{1}^{(2)}$ & & $\kappa_{2}^{(1)}$ & $\kappa_{2}^{(2)}$ \\
$\kappa_{2}^{(0)}$ & & & & \\
$\kappa_{3}^{(-2)}$ & $\kappa_{3}^{(-1)}$ & $\kappa_{3}^{(0)}$ & $\kappa_{4}^{(0)}$ & \\
$\kappa_{4}^{(-1)}$ & & & & \\
$\kappa_{5}^{(-4)}$ & $\kappa_{5}^{(-3)}$ & $\kappa_{5}^{(-2)}$ & & \\
$\kappa_{6}^{(-3)}$ & $\kappa_{6}^{(-2)}$ & $\kappa_{6}^{(-1)}$ & & \\
$\kappa_{7}^{(-2)}$ & & & $\kappa_{7}^{(-1)}$ & $\kappa_{7}^{(0)}$
\end{tabular}

Upon substitution of these expressions in the perturbative contributions of the energy we have

$$
\begin{aligned}
& E_{0}^{(1)}=O(\beta), \\
& E_{0}^{(2)}=-\epsilon_{0}^{2} \kappa_{2}^{(0)}+O(\beta), \\
& E_{0}^{(3)}=\epsilon_{0}^{3} \frac{\kappa_{1}^{(1)} \kappa_{3}^{(-2)}-\kappa_{4}^{(-1)}}{\beta} \\
& \quad+\epsilon_{0}^{3}\left[\kappa_{1}^{(2)} \kappa_{3}^{(-2)}+\kappa_{1}^{(1)} \kappa_{3}^{(-1)}-\kappa_{4}^{(0)}\right]+O(\beta),
\end{aligned}
$$


and

$$
\begin{aligned}
& E_{0}^{(4)}=\eta_{4 a}\left(\frac{\epsilon_{0}^{4}}{\beta^{2}}-4 \epsilon_{0}^{3}\right)+\eta_{4 b} \frac{\epsilon_{0}^{4}}{\beta}+\eta_{4 c} \epsilon_{0}^{3} \\
& \quad+\eta_{4 d} \epsilon_{0}^{4}+O(\beta),
\end{aligned}
$$

where

$$
\begin{aligned}
& \eta_{4 a} \equiv \\
&\left(-\kappa_{5}^{(-4)}\left(\kappa_{1}^{(1)}\right)^{2}+2 \kappa_{6}^{(-3)} \kappa_{1}^{(1)}+\kappa_{2}^{(0)} \kappa_{3}^{(-2)}-\kappa_{7}^{(-2)}\right), \\
& \eta_{4 b} \equiv\left(-\kappa_{5}^{(-3)}\left(\kappa_{1}^{(1)}\right)^{2}-2 \kappa_{1}^{(2)} \kappa_{5}^{(-4)} \kappa_{1}^{(1)}+2 \kappa_{6}^{(-2)} \kappa_{1}^{(1)}\right. \\
&\left.\quad+\kappa_{2}^{(1)} \kappa_{3}^{(-2)}+\kappa_{2}^{(0)} \kappa_{3}^{(-1)}+2 \kappa_{1}^{(2)} \kappa_{6}^{(-3)}-\kappa_{7}^{(-1)}\right), \\
& \eta_{4 c} \equiv 2\left(\left(\kappa_{2}^{(0)}\right)^{2}+2 \kappa_{1}^{(1)}\left(\kappa_{4}^{(-1)}-\kappa_{1}^{(1)} \kappa_{3}^{(-2)}\right)\right), \\
& \eta_{4 d} \equiv\left(-\kappa_{5}^{(-2)}\left(\kappa_{1}^{(1)}\right)^{2}-2 \kappa_{1}^{(3)} \kappa_{5}^{(-4)} \kappa_{1}^{(1)}\right. \\
&-2 \kappa_{1}^{(2)} \kappa_{5}^{(-3)} \kappa_{1}^{(1)}+2 \kappa_{6}^{(-1)} \kappa_{1}^{(1)}+\kappa_{2}^{(2)} \kappa_{3}^{(-2)} \\
&+\kappa_{2}^{(1)} \kappa_{3}^{(-1)}+\kappa_{2}^{(0)} \kappa_{3}^{(0)}-\left(\kappa_{1}^{(2)}\right)^{2} \kappa_{5}^{(-4)}+2 \kappa_{1}^{(3)} \kappa_{6}^{(-3)} \\
&\left.+2 \kappa_{1}^{(2)} \kappa_{6}^{(-2)}-\kappa_{7}^{(0)}\right) .
\end{aligned}
$$

Observe that the potentially divergent terms in $E_{0}^{(3)}$ and $E_{0}^{(4)}$ only depend on the contributions stemming from the longitudinal excitations. While it was already proved in Ref. [9] that $E_{0}^{(3)}$ is finite for $\beta \rightarrow 0^{+}$, as it can be checked explicitly using the results in Appendix B of Ref. [14], it is straightforward to verify that $\eta_{4 a}=\eta_{4 b}=0$. Therefore $E_{0}^{(4)}$ is finite for $\beta \rightarrow 0^{+}$, as expected.

Using the expressions in Appendix of Ref. [14] we have

$$
\eta_{4 c}=\frac{2}{b^{4}}\left(\int \mathrm{d} x \mathrm{~d} y \cos ^{2} \frac{\pi y}{b} \sigma(x, y)\right)^{4}
$$

and

$$
\eta_{4 d}=\eta_{4 d}^{\|}+\eta_{4 d}^{\perp}
$$

where $\eta_{4 d}^{\|}$contains only contributions from longitudinal modes while $\eta_{4 d}^{\perp}$ contains contributions also from transversal modes.

Their explicit expressions are $\mathbb{I}$

$$
\begin{gathered}
\eta_{4 d}^{\|}=\frac{1}{b^{4}} \int \mathrm{d} x_{1} \mathrm{~d} y_{1} \int \mathrm{d} x_{2} \mathrm{~d} y_{2} x_{1}\left(2 x_{2}-x_{1}\right) \sigma\left(\boldsymbol{x}_{1}\right) \sigma\left(\boldsymbol{x}_{2}\right) \\
\quad \times \cos ^{2}\left(\frac{\pi y_{1}}{b}\right) \cos ^{2}\left(\frac{\pi y_{2}}{b}\right)\left(\int \mathrm{d} x_{3} \mathrm{~d} y_{3} \cos ^{2}\left(\frac{\pi y_{3}}{b}\right)\right. \\
\left.\quad \times \sigma\left(\boldsymbol{x}_{3}\right)\right)^{2}-\frac{2}{b^{4}} \int \mathrm{d} x_{1} \mathrm{~d} y_{1} \int \mathrm{d} x_{2} \mathrm{~d} y_{2} \int \mathrm{d} x_{3} \mathrm{~d} y_{3} \\
\quad \times\left|x_{1}-x_{2}\right|\left|x_{2}-x_{3}\right| \sigma\left(\boldsymbol{x}_{1}\right) \sigma\left(\boldsymbol{x}_{2}\right) \sigma\left(\boldsymbol{x}_{3}\right) \cos ^{2}\left(\frac{\pi y_{1}}{b}\right)
\end{gathered}
$$

I The expression for $g_{2}^{(0,0)}\left(x_{1}, y_{1}, x_{2}, y_{2}\right)$ is reported in Appendix A of Ref. [14].

$$
\begin{aligned}
& \times \cos ^{2}\left(\frac{\pi y_{2}}{b}\right) \cos ^{2}\left(\frac{\pi y_{3}}{b}\right) \times \int \mathrm{d} x_{4} \mathrm{~d} y_{4} \sigma\left(\boldsymbol{x}_{4}\right) \\
& \times \cos ^{2}\left(\frac{\pi y_{4}}{b}\right)-\frac{1}{b^{4}}\left(\int \mathrm{d} x_{1} \mathrm{~d} y_{1} \int \mathrm{d} x_{2} \mathrm{~d} y_{2}\right. \\
& \left.\times\left|x_{1}-x_{2}\right| \cos ^{2}\left(\frac{\pi y_{1}}{b}\right) \cos ^{2}\left(\frac{\pi y_{2}}{b}\right) \sigma\left(\boldsymbol{x}_{1}\right) \sigma\left(\boldsymbol{x}_{2}\right)\right)^{2}, \\
& \eta_{4 d}^{\perp}=\frac{1}{b^{3}} \int \mathrm{d} x_{1} \mathrm{~d} y_{1} \int \mathrm{d} x_{2} \mathrm{~d} y_{2} \int \mathrm{d} x_{3} \mathrm{~d} y_{3} \int \mathrm{d} x_{4} \mathrm{~d} y_{4} \\
& \times \sigma\left(\boldsymbol{x}_{1}\right) \sigma\left(\boldsymbol{x}_{2}\right) \sigma\left(\boldsymbol{x}_{3}\right) \sigma\left(\boldsymbol{x}_{4}\right) \cos \left(\frac{\pi y_{1}}{b}\right) \cos \left(\frac{\pi y_{2}}{b}\right) \\
& \times \cos ^{2}\left(\frac{\pi y_{3}}{b}\right) \cos ^{2}\left(\frac{\pi y_{4}}{b}\right)\left(2\left|x_{1}-x_{3}\right|+\left|x_{3}-x_{4}\right|\right) \\
& \times g_{2}^{(0,0)}\left(\boldsymbol{x}_{1}, \boldsymbol{x}_{2}\right)-\frac{2}{b^{2}} \int \mathrm{d} x_{1} \mathrm{~d} y_{1} \int \mathrm{d} x_{2} \mathrm{~d} y_{2} \\
& \times \int \mathrm{d} x_{3} \mathrm{~d} y_{3} \cos \left(\frac{\pi y_{1}}{b}\right) \cos \left(\frac{\pi y_{3}}{b}\right) g_{2}^{(0,0)}\left(\boldsymbol{x}_{1}, \boldsymbol{x}_{2}\right) \\
& \times g_{2}^{(0,0)}\left(\boldsymbol{x}_{2}, \boldsymbol{x}_{3}\right) \sigma\left(\boldsymbol{x}_{1}\right) \sigma\left(\boldsymbol{x}_{2}\right) \sigma\left(\boldsymbol{x}_{3}\right) \\
& \times \int \mathrm{d} x_{4} \mathrm{~d} y_{4} \cos ^{2}\left(\frac{\pi y_{4}}{b}\right) \sigma\left(\boldsymbol{x}_{4}\right) \\
& \quad-\frac{1}{b^{2}}\left(\int \mathrm{d} x_{1} \mathrm{~d} y_{1} \int \mathrm{d} x_{2} \mathrm{~d} y_{2} \cos \left(\frac{\pi y_{1}}{b}\right)\right. \\
& \left.\times \cos \left(\frac{\pi y_{2}}{b}\right) g_{2}^{(0,0)}\left(\boldsymbol{x}_{1}, \boldsymbol{x}_{2}\right) \sigma\left(\boldsymbol{x}_{1}\right) \sigma\left(\boldsymbol{x}_{2}\right)\right)^{2} .
\end{aligned}
$$

We may write the perturbative formulae obtained above in a more compact form as

$$
\begin{aligned}
& E_{0}^{(2)}=-\frac{\pi^{2}}{b^{2}} \Delta_{2}^{2} \\
& E_{0}^{(3)}=-2 \frac{\pi^{2}}{b^{2}} \Delta_{2}\left(\Lambda_{1}-\Delta_{3}\right), \\
& E_{0}^{(4)}=-\frac{\pi^{2}}{b^{2}}\left[-2 \Delta_{2}^{4}-\Delta_{2}^{2} \Delta_{4}+2 \Delta_{2} \Delta_{5}+\Delta_{3}^{2}-2 \Lambda_{2}\right. \\
& \left.\quad-\Delta_{3} \Lambda_{1}+2 \Delta_{2} \Lambda_{3}+\Lambda_{1}^{2}\right],
\end{aligned}
$$

where we have introduced the definitions

$$
\begin{aligned}
\Delta_{1} & \equiv \frac{\pi}{b^{2}} \int \mathrm{d} x \mathrm{~d} y \sigma(\boldsymbol{x}), \\
\Delta_{2} & \equiv \frac{\pi}{b^{2}} \int \mathrm{d} x \mathrm{~d} y \sigma(\boldsymbol{x}) \cos ^{2} \frac{\pi y}{b}, \\
\Delta_{3} & \equiv \frac{\pi^{3}}{b^{5}} \int \mathrm{d} x_{1} \mathrm{~d} y_{1} \int \mathrm{d} x_{2} \mathrm{~d} y_{2} \sigma\left(\boldsymbol{x}_{1}\right) \sigma\left(\boldsymbol{x}_{2}\right)\left|x_{1}-x_{2}\right| \\
& \times \cos ^{2} \frac{\pi y_{1}}{b} \cos ^{2} \frac{\pi y_{2}}{b}, \\
\Delta_{4} & \equiv \frac{\pi^{4}}{b^{6}} \int \mathrm{d} x_{1} \mathrm{~d} y_{1} \int \mathrm{d} x_{2} \mathrm{~d} y_{2} \sigma\left(\boldsymbol{x}_{1}\right) \sigma\left(\boldsymbol{x}_{2}\right) x_{1}\left(2 x_{2}-x 1\right) \\
& \times \cos ^{2} \frac{\pi y_{1}}{b} \cos ^{2} \frac{\pi y_{2}}{b}, \\
\Delta_{5} & \equiv \frac{\pi^{5}}{b^{8}} \int \mathrm{d} x_{1} \mathrm{~d} y_{1} \int \mathrm{d} x_{2} \mathrm{~d} y_{2} \int \mathrm{d} x_{3} \mathrm{~d} y_{3} \sigma\left(\boldsymbol{x}_{1}\right) \sigma\left(\boldsymbol{x}_{2}\right) \\
& \times \sigma\left(\boldsymbol{x}_{3}\right)\left|x_{1}-x_{2}\right|\left|x_{2}-x_{3}\right| \cos ^{2} \frac{\pi y_{1}}{b} \cos ^{2} \frac{\pi y_{2}}{b} \cos ^{2} \frac{\pi y_{3}}{b},
\end{aligned}
$$




$$
\begin{aligned}
\Lambda_{1} & \equiv \frac{\pi^{3}}{b^{4}} \int \mathrm{d} x_{1} \mathrm{~d} y_{1} \int \mathrm{d} x_{2} \mathrm{~d} y_{2} \sigma\left(\boldsymbol{x}_{1}\right) \sigma\left(\boldsymbol{x}_{2}\right) \cos \frac{\pi y_{1}}{b} \\
& \times \cos \frac{\pi y_{2}}{b} g_{2}^{(0,0)}\left(\boldsymbol{x}_{1}, \boldsymbol{x}_{2}\right), \\
\Lambda_{2} & \equiv \frac{\pi^{6}}{b^{9}} \int \mathrm{d} x_{1} \mathrm{~d} y_{1} \int \mathrm{d} x_{2} \mathrm{~d} y_{2} \int \mathrm{d} x_{3} \mathrm{~d} y_{3} \int \mathrm{d} x_{4} \mathrm{~d} y_{4} \\
& \times \sigma\left(\boldsymbol{x}_{1}\right) \sigma\left(\boldsymbol{x}_{2}\right) \sigma\left(\boldsymbol{x}_{3}\right) \sigma\left(\boldsymbol{x}_{4}\right)\left|x_{1}-x_{3}\right| \cos \frac{\pi y_{1}}{b} \cos \frac{\pi y_{2}}{b} \\
& \times \cos ^{2} \frac{\pi y_{3}}{b} \cos ^{2} \frac{\pi y_{4}}{b} g_{2}^{(0,0)}\left(\boldsymbol{x}_{1}, \boldsymbol{x}_{2}\right), \\
\Lambda_{3} & \equiv \frac{\pi^{5}}{b^{6}} \int \mathrm{d} x_{1} \mathrm{~d} y_{1} \int \mathrm{d} x_{2} \mathrm{~d} y_{2} \int \mathrm{d} x_{3} \mathrm{~d} y_{3} \sigma\left(\boldsymbol{x}_{1}\right) \sigma\left(\boldsymbol{x}_{2}\right) \\
& \times \sigma\left(\boldsymbol{x}_{3}\right) \cos \frac{\pi y_{1}}{b} \cos \frac{\pi y_{3}}{b} g_{2}^{(0,0)}\left(\boldsymbol{x}_{1}, \boldsymbol{x}_{2}\right) g_{2}^{(0,0)}\left(\boldsymbol{x}_{2}, \boldsymbol{x}_{3}\right),
\end{aligned}
$$

where $\Delta_{1}$ is the total extra mass of the inhomogeneous waveguide.

The energy up to fourth order can then be arranged in the form

$$
\begin{aligned}
& \Delta E_{0} \approx E_{0}^{(2)}+E_{0}^{(3)}+E_{0}^{(4)}= \\
& -\frac{\pi^{2}}{b^{2}}\left\{\left(\Delta_{2}+\left(\Lambda_{1}-\Delta_{3}\right)^{2}\right)^{2}+\Gamma\right\},
\end{aligned}
$$

where

$$
\begin{aligned}
\Gamma & \equiv\left[-2 \Delta_{2}^{4}+\Delta_{2} \Delta_{3}-\Delta_{2}^{2} \Delta_{4}+2 \Delta_{2} \Delta 5-\Delta_{3} \Lambda_{1}\right. \\
& \left.-2 \Lambda_{2}+2 \Delta_{2} \Lambda_{3}\right] .
\end{aligned}
$$

When we apply the formulae above to the solvable model discussed in Ref. [9] we obtain

$$
E_{0}^{(4)}=\frac{\sigma^{4}\left(90 \pi^{6} b^{2} \delta^{4}-23 \pi^{8} \delta^{6}\right)}{720 b^{8}}
$$

which reproduces the exact expression for the fourth order contribution reported in Ref. [9].

\section{A solvable model}

The case where the second and third order contributions vanish is particularly interesting and it deserves a detailed discussion. This situation is analogous to the case discussed by Exner and Vugalter in Ref. [8] for a uniform, weakly deformed, waveguide.

As previously observed in Ref. [9] this occurs when the density obeys the property

$$
\int \mathrm{d} x \mathrm{~d} y \cos ^{2} \frac{\pi y}{b} \sigma(\boldsymbol{x})=0 .
$$

In this limit the general formulae obtained in the previous section reduce to

$$
\begin{aligned}
\eta_{4 c} & =0 \\
\eta_{4 d}^{\|} & =-\frac{1}{b^{4}}\left(\int \mathrm{d} x_{1} \mathrm{~d} y_{1} \int \mathrm{d} x_{2} \mathrm{~d} y_{2}\left|x_{1}-x_{2}\right| \cos ^{2}\left(\frac{\pi y_{1}}{b}\right)\right. \\
\times & \left.\cos ^{2}\left(\frac{\pi y_{2}}{b}\right) \sigma\left(\boldsymbol{x}_{1}\right) \sigma\left(\boldsymbol{x}_{2}\right)\right)^{2}, \\
\eta_{4 d}^{\perp} & =-\frac{1}{b^{2}}\left[\int \mathrm{d} x_{1} \mathrm{~d} y_{1} \int \mathrm{d} x_{2} \mathrm{~d} y_{2} \cos \left(\frac{\pi y_{1}}{b}\right)\right.
\end{aligned}
$$

$$
\left.\times \cos \left(\frac{\pi y_{2}}{b}\right) g_{2}^{(0,0)}\left(\boldsymbol{x}_{1}, \boldsymbol{x}_{2}\right) \sigma\left(\boldsymbol{x}_{1}\right) \sigma\left(\boldsymbol{x}_{2}\right)\right]^{2}
$$

and the energy of the fundamental mode falls below the threshold of the continuum, signalling that the corresponding eigenfunction is localized in the region of the heterogeneity.

To test this prediction, we consider a solvable model, represented by an infinite heterogeneous waveguide, parallel to the horizontal axis and obeying the Dirichlet boundary conditions on $y= \pm b / 2$ (see Fig. 1).

The density is

$$
\Sigma(x)=\left\{\begin{array}{l}
1+\sigma_{1},|x|<\delta_{1} / 2 \\
1+\sigma_{2}, \delta_{1} / 2<|x|<\delta_{2} / 2, \\
1,|x|>\delta_{2} / 2
\end{array}\right.
$$

where $\delta_{2} \geq \delta_{1} \geq 0$ (for $\sigma_{1}=\sigma_{2}$ this problem reduces to the one discussed in Ref. [9]).

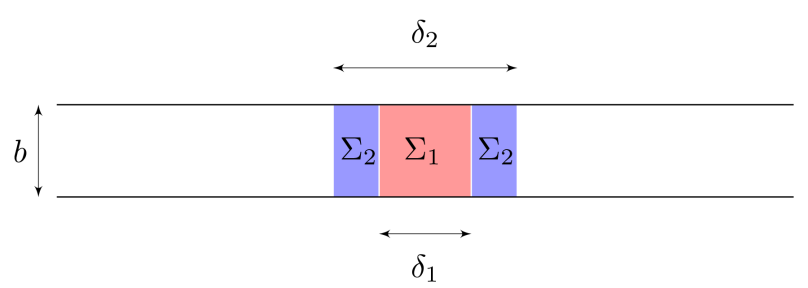

Fig. 1. Heterogeneous waveguide with three regions of different density.

We look for the solution to the Helmholtz equation

$$
-\Delta \Psi(x, y)=E \Sigma(x) \Psi(x, y)
$$

in the form

$$
\begin{aligned}
& \Psi(x, y)=\sqrt{\frac{2}{b}} \sin \frac{\pi n(y+b / 2)}{b} \\
& \quad \times\left\{\begin{array}{l}
A_{1} \cos \left(p_{1} x\right),|x|<\delta_{1} / 2 \\
A_{2} \cos \left(p_{2} x+q_{2}\right), \delta_{1} / 2<|x|<\delta_{2} / 2, \\
A_{3} \mathrm{e}^{-\alpha|x|},|x|>\delta_{2} / 2
\end{array}\right.
\end{aligned}
$$

where the unknown coefficients are to be obtained enforcing the continuity of the solution and its derivative at $x=\delta_{1} / 2$ and $x=\delta_{2} / 2$ (since the solution for the fundamental mode must be even, the matching at $x=-\delta_{1} / 2$ and $x=-\delta_{2} / 2$ is automatic). Since we are interested only in the fundamental mode we may set $n=1$.

By asking that $\Psi(x, y)$ be a solution to the Helmholtz equation on each region we obtain

$$
\begin{aligned}
& p_{1}=\sqrt{k^{2}\left(1+\sigma_{1}\right)-\pi^{2} / b^{2}}, \\
& p_{2}=\sqrt{k^{2}\left(1+\sigma_{2}\right)-\pi^{2} / b^{2}}, \\
& \alpha=\sqrt{\pi^{2} / b^{2}-k^{2}}
\end{aligned}
$$

From the matching of the solutions we obtain the transcendental equations

$$
A_{1} \cos \left(\frac{\delta_{1} p_{1}}{2}\right)=A_{2} \cos \left(\frac{\delta_{1} p_{2}}{2}+q_{2}\right)
$$




$$
\begin{aligned}
& A_{1} p_{1} \sin \left(\frac{\delta_{1} p_{1}}{2}\right)=A_{2} p_{2} \sin \left(\frac{\delta_{1} p_{2}}{2}+q_{2}\right) \\
& A_{3} \mathrm{e}^{-\alpha \delta_{2} / 2}=A_{2} \cos \left(\frac{\delta_{2} p_{2}}{2}+q_{2}\right)-\alpha A_{3} \mathrm{e}^{-\alpha \delta_{2} / 2}= \\
& A_{2} p_{2} \sin \left(\frac{\delta_{2} p_{2}}{2}+q_{2}\right)
\end{aligned}
$$

which can be reduced to

$$
\begin{aligned}
& \frac{p_{1}}{p_{2}} \tan \left(\frac{\delta_{1} p_{1}}{2}\right)=\tan \left(\frac{\delta_{1} p_{2}}{2}+q_{2}\right), \\
& \alpha=p_{2} \tan \left(\frac{\delta_{1} p_{2}}{2}+q_{2}\right),
\end{aligned}
$$

after eliminating the amplitudes.

We look for a solution to these equations, in the limit of weak inhomogeneities: to perform the appropriate expansion in the density we introduce a parameter $\eta$, to keep track of the order of the expansion and make the substitutions $\sigma_{i} \rightarrow \eta \sigma_{i}$ (at the end of the calculation we will let $\eta \rightarrow 1$ ).

We also express $k$ and $q_{2}$ in terms of appropriate power series

$$
q_{2}=\sum_{n=0}^{\infty} c_{n} \eta^{n / 2}, \quad k=\sqrt{\frac{\pi^{2}}{b^{2}}+\sum_{n=1}^{\infty} \kappa_{n} \eta^{n}} .
$$

After substituting these expressions in Eqs. (30) and (31) one obtains the explicit expression for the lowest eigenvalue

$$
\begin{aligned}
E_{0} & =k^{2}=\frac{\pi^{2}}{b^{2}}-\frac{\pi^{4}\left(\delta_{1}\left(\sigma_{1}-\sigma_{2}\right)+\delta_{2} \sigma_{2}\right)^{2}}{4 b^{4}} \\
& +\frac{\pi^{6}\left(\delta_{1}\left(\sigma_{1}-\sigma_{2}\right)+\delta_{2} \sigma_{2}\right)}{24 b^{6}}\left(\delta_{1}^{3}\left(2 \sigma_{1}^{2}-3 \sigma_{2} \sigma_{1}+\sigma_{2}^{2}\right)\right. \\
& \left.+3 \delta_{2}^{2} \delta_{1}\left(\sigma_{1}-\sigma_{2}\right) \sigma_{2}+2 \delta_{2}^{3} \sigma_{2}^{2}\right) \\
& +\frac{\sigma_{1}^{4}\left(90 \pi^{6} b^{2} \delta_{1}^{4}-23 \pi^{8} \delta_{1}^{6}\right)}{720 b^{8}} \\
& +\frac{\pi^{6} \delta_{1}^{3}\left(\delta_{1}-\delta_{2}\right) \sigma_{2} \sigma_{1}^{3}}{240 b^{8}} \\
& \times\left(\pi^{2}\left(26 \delta_{1}^{2}+15 \delta_{2} \delta_{1}+5 \delta_{2}^{2}\right)-120 b^{2}\right) \\
& -\frac{\pi^{6} \delta_{1}^{2}\left(\delta_{1}-\delta_{2}\right)^{2} \sigma_{2}^{2} \sigma_{1}^{2}}{576 b^{8}} \\
& \times\left(\pi^{2}\left(79 \delta_{1}^{2}+86 \delta_{2} \delta_{1}+51 \delta_{2}^{2}\right)-432 b^{2}\right) \\
+ & \frac{\pi^{6} \delta_{1}\left(\delta_{1}-\delta_{2}\right)^{3} \sigma_{2}^{3} \sigma_{1}}{480 b^{8}} \\
& \times\left(\pi^{2}\left(37 \delta_{1}^{2}+56 \delta_{2} \delta_{1}+47 \delta_{2}^{2}\right)-240 b^{2}\right) \\
& +\frac{\pi^{8}\left(\delta_{1}-\delta_{2}\right)^{4}\left(47 \delta_{1}^{2}+86 \delta_{2} \delta_{1}+92 \delta_{2}^{2}\right) \sigma_{2}^{4}}{2880 b^{8}} \\
& \frac{\pi^{6} 360 b^{2}\left(\delta_{2} \sigma_{2}-\delta_{1} \sigma_{2}\right)^{4}}{288 b^{8}}+\ldots \\
&
\end{aligned}
$$

subject to the condition

$$
\delta_{1}\left(\sigma_{1}-\sigma_{2}\right)+\delta_{2} \sigma_{2} \geq 0 .
$$

In particular it is interesting to consider the case $\sigma_{1}=\frac{\left(\delta_{1}-\delta_{2}\right) \sigma_{2}}{\delta_{1}}$, corresponding to a waveguide where the heterogeneity averages to zero; in this case the energy reduces to

$$
\begin{aligned}
E_{0} & =\frac{\pi^{2}}{b^{2}}-\frac{\pi^{8}\left(\delta_{1}-\delta_{2}\right)^{4} \delta_{2}^{2} \sigma_{2}^{4}}{576 b^{8}} \\
& +\frac{\pi^{10}\left(\delta_{1}-3 \delta_{2}\right)\left(\delta_{1}-\delta_{2}\right)^{5} \delta_{2}^{2} \sigma_{2}^{5}}{5760 b^{10}}+\ldots
\end{aligned}
$$

where we have reported the fifth order as well (we do not report the fifth order for the general case, because of its length).

For this model the perturbative formulae derived in the previous section up to fourth order yield

$$
\begin{aligned}
& E_{0}^{(\text {pert })}=\frac{\pi^{2}}{b^{2}}-\frac{\pi^{8}}{b^{12}}\left(\int \mathrm{d} x_{1} \mathrm{~d} y_{1} \int \mathrm{d} x_{2} \mathrm{~d} y_{2}\left|x_{1}-x_{2}\right|\right. \\
& \left.\quad \times \cos ^{2}\left(\frac{\pi y_{1}}{b}\right) \cos ^{2}\left(\frac{\pi y_{2}}{b}\right) \sigma\left(\boldsymbol{x}_{1}\right) \sigma\left(\boldsymbol{x}_{2}\right)\right)^{2}= \\
& \frac{\pi^{2}}{b^{2}}-\frac{\pi^{8}\left(\delta_{1}-\delta_{2}\right)^{4} \delta_{2}^{2} \sigma_{2}^{4}}{576 b^{8}},
\end{aligned}
$$

which confirms the exact result of Eq. (32).

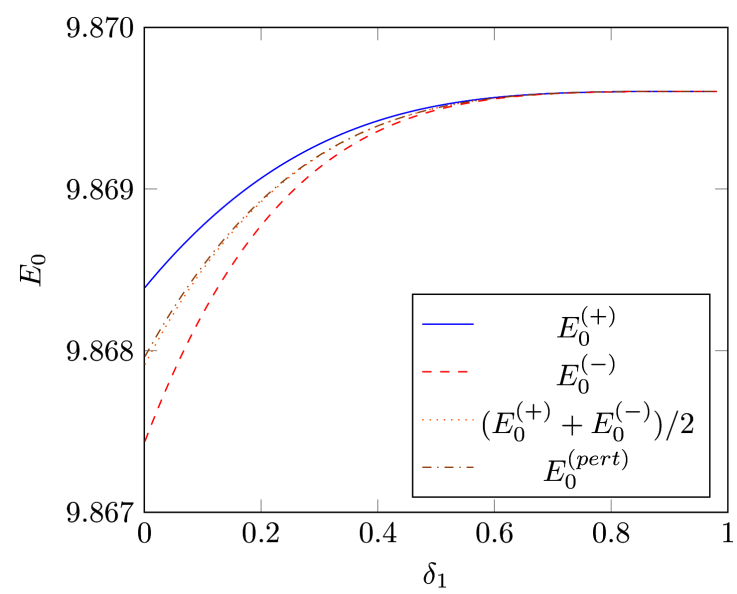

Fig. 2. Energy of the fundamental mode of the solvable model, for the case $\delta_{2}=1, b=1, \sigma_{1}=\frac{\left(\delta_{1}-\delta_{2}\right) \sigma_{2}}{\delta_{1}}$ and $\left|\sigma_{2}\right|=1 / 10$.

In Fig. 2 we plot the energy of the fundamental mode for the case $\delta_{2}=1, b=1, \sigma_{1}=\frac{\left(\delta_{1}-\delta_{2}\right) \sigma_{2}}{\delta_{1}}$, as a function of $\delta_{1} \cdot E_{0}^{( \pm)}$correspond to the numerical solution of Eqs. (30) and (31) for $\sigma_{2}= \pm 1 / 10$, while $E_{0}^{(\text {pert })}$ is the expression of Eq. (32). Notice that, while $E_{0}^{( \pm)}$departs from the perturbative formula $E_{0}^{(\text {pert })}$ for $\delta_{1} \rightarrow 0$, the average of the two is remarkably close to $E_{0}^{(p e r t)}$. This is consistent with the form of the fifth order contribution reported in Eq. (32), which changes sign in the two cases. 


\section{Conclusions}

In this paper we have applied the method described in Ref. [9] to calculate the fourth order perturbative correction to the energy of the ground state of an infinite waveguide, with a small heterogeneity localized around a given internal point.

We may summarize the main results with the following points:

- the expression for $E_{0}^{(4)}$ is finite for $\beta \rightarrow 0^{+}$, as expected (notice that, as the perturbative order increases there are more potentially divergent terms; for instance, while the third order only contains a term which diverges as $1 / \beta$, the fourth order contains a term that diverges as $1 / \beta^{2}$ as well);

- for waveguides where the second and third orders vanish, there may still be a bound state and the energy gap scales as the fourth power in the density (consistent with the observation made in Ref. [8] for the problem of the deformed waveguide);

- the exact results for two solvable models are reproduced to fourth order;

- the perturbative scheme adopted in this paper and in Ref. [9] is fully consistent, and it could be used to obtain higher order contributions.

In our view the calculation of the fourth order perturbative correction carried out in this paper is important for several reasons: on one hand, in special cases, such as the broken strip studied in Ref. [12], the leading correction to the unperturbed energy depends on this contribution and therefore it must be taken into account, in second place, the calculation of higher perturbative orders opens the door to the possibility of extending the results to a nonperturbative regime (large densities and large deformations) and of obtaining analytical results in cases where usually only numerical results are available. One example of this is the calculation performed by Amore and Fernández in Ref. [15], who applied the method of Gat and Rosenstein to the case of weakly attractive potentials in one dimensions up to sixth order. The perturbative formulae obtained there were resumed using a Pade approximant and applied to the not exactly solvable problem of a Gaussian well, finding excellent agreement with the numerical result. Finally, it is also important to underline that the present calculation, as the one of Ref. [15], prove the consistency of the method of Ref. [10] when applied to higher orders.

\section{Acknowledgments}

This research was supported by the Sistema Nacional de Investigadores (México). The figures were produced using Tikz [16].

\section{References}

[1] P. Exner, P. Seba, J. Math. Phys. 30, 2574 (1989).

[2] J. Goldstone, R.L. Jaffe, Phys. Rev. B 45, 14100 (1992).

[3] R.L. Schult, D.G. Ravenhall, H.W. Wyld, Phys. Rev. $B$ 39, 5476 (1989).

[4] S. Qu, M.R. Geller, Phys. Rev. B 70, 085414 (2004).

[5] F. Ursell, Proc. Camb. Philos. Soc. 47, 348 (1951).

[6] F. Ursell, Proc. R. Soc. Lond. A 214, 79 (1952).

[7] W. Bulla, F. Gesztesy, W. Renger, B. Simon, Proc. Am. Math. Soc. 125, 1487 (1997).

[8] P. Exner, S.A. Vugalter, Lett. Math. Phys. 39, 59 (1997).

[9] P. Amore, F.M. Fernández, C.P. Hofmann, Europ. Phys. J. B 89, 163 (2016).

[10] G. Gat, B. Rosenstein, Phys. Rev. Lett. 70, 5 (1993).

[11] P. Amore, J.P. Boyd, F.M. Fernández, M. Jacobo, P. Zhevandrov, The Anziam Journal 1, 15 (2017).

[12] Y. Avishai, D. Bessis, B.G. Giraud, G. Mantica, Phys. Rev. B 44, 233101 (1991).

[13] P. Amore, J. Math. Phys. 51, 052105 (2010).

[14] P. Amore, arXiv:1601.02470, 2016.

[15] P. Amore, F.M. Fernández, Ann. Phys. 378, 253 (2017).

[16] Till Tantau, The TikZ and PGF Packages, Manual for version 3.0.0. 